\title{
Preface to the special issues of the Twelfth European Wave and Tidal Energy Conference (EWTEC 2017)
}

\author{
A.W. Lewis
}

$\mathrm{T}$ HE twelfth conference of the European Wave and Tidal Energy Conference series was held at the verdant campus of University College Cork, Ireland, from Sunday 27th August to Saturday 1st September 2017. EWTEC 2017 was brought to Ireland as it has the best resource anywhere on the planet for wave energy and to a lesser extent for tidal stream energy and the Government support for marine renewable energy is second to none. This Conference was an essential component in support of the development of the exciting and emerging renewable marine energy industry. It brought together the world leading academics, researchers and industry developers together with a large cohort of young researchers. EWTEC2017 also had an exhibition where leading companies and research centres presented ongoing developments. There were also a number of international workshops related to specific themes of marine renewable energy development.

At EWTEC2017 the most up-to-date results from innovative research within both academia and industry were presented. The Conference had over 530 attendees from at least 25 countries, with the largest number of papers ever submitted to the proceedings and delivered through 336 oral presentations and 30 poster presentations over the four days. This is the second time the Conference was held in Cork with the previous fifth Conference in the EWTEC series held in 2003 when only 120 delegates attended.
The thematic tracks of the conference covered fundamental hydrodynamics, environmental impact assessment, economics, social and policy implications of wave and tidal energy. The tracks also included numerical modelling and thorough engineering studies, all specifically dedicated to the development of wave and tidal energy conversion devices. As the Chair of EWTEC 2017, I have asked the international peer-reviewers, to nominate the best papers in their respective tracks. As a member of the Editorial Board of the International Marine Energy Journal (IMEJ), together with Prof A.S. Bahaj, the Editor-in-Chief, we have decided to dedicate a special issue of the journal to present the best papers selected from EWTEC2017 conference.

The first two issues of IMEJ are dedicated to top rated papers reviewed by at least two peer reviewers. These volumes represent the first special IMEJ issues of this kind and I hope that this achievement will establish a tradition, every two years, to follow the biannual scheduling of EWTEC. I found this combined approach of the rigorous reviewing processes and paper selection to be highly beneficial for the whole marine energy scientific community. In my view such combination provides the most effective way to efficiently disseminate the advances in marine energy research and development to the global community.

Prof Tony Lewis Chair, EWTEC 2017
CA.W. Lewis 2018. This is an open access article distributed under the terms of the Creative Commons Attribution 4.0 licence (CC BY http://creativecommons.org/licenses/by/4.0/). Unrestricted use (including commercial), distribution and reproduction is permitted provided that credit is given to the original author(s) of the work, including a URI or hyperlink to the work, this public license and a copyright notice.

A.W. Lew is is the Chair of EWTEC 2017 and Emeritus Beaufort Professor at University College Cork, T12 K8AF, Ireland (e-mail t.lewis@ucc.ie) 